\title{
Mathematics and the Sciences
}

\section{Introduction}

The principal thrust of this essay is to describe the current state of interaction between mathematics and the sciences and to relate the trends to the historical development of mathematics as an intellectual discipline and of the sciences as they have developed since the seventeenth century. This story is interesting in the context of the history of present-day mathematics because it represents a shift in the preconceptions and stereotypes of both mathematicians and scientists since World War II.

The notion that significant mathematical and scientific advances are closely interwoven is not particularly new. The opposing notion (associated, with whatever degree of justice, with the name of Bourbaki) was never as fashionable, at least among working mathematicians, as in the two decades immediately after World War II. The situation has changed significantly during the past decade and had begun turning even earlier. It turned not only among mathematicians, but even more significantly in such sciences as physics. The frontier of mathematical advance was seen again to be in forceful interaction with the basic problems and needs of scientific advance.

This is an essay on significant trends in mathematical practice. The relations between the history and philosophy of mathematics as usually conceived and mathematical practice have often been very ambiguous. In part, this has resulted from the efforts of some historians and philosophers to impose a framework of preconceptions upon mathematical practice that had little to do with the latter. In part, however, it resulted from the diversity of mathematical practice, to lags in its perception, and to the complexity of viewpoints embedded in that practice.

Let me preface this account with two statements by great American mathematicians of an earlier period who put the case in a sharp form. 
The first is John von Neumann in a 1945 essay titled "The Mathematician": 1

Most people, mathematicians and others, will agree that mathematics is not an empirical science, or at least that it is practiced in a manner which differs in several decisive respects from the techniques of the empirical sciences. And, yet, its development is very closely linked with the natural sciences. One of its main branches, geometry, actually started as a natural, empirical science. Some of the best inspirations of modern mathematics (I believe, the best ones) clearly originated in the natural sciences. The methods of mathematics pervade and dominate the "theoretical" divisions of the natural sciences. In modern empirical sciences it has become more and more a major criterion of success whether they have become accessible to the mathematical method or to the near-mathematical methods of physics. Indeed, throughout the natural sciences an unbroken chain of successive pseudomorphoses, all of them pressing toward mathematics, and almost identified with the idea of scientific progress, has become more and more evident. Biology becomes increasingly pervaded by chemistry and physics, chemistry by experimental and theoretical physics, and physics by very mathematical forms of theoretical physics.

The second is Norbert Wiener in a 1938 essay titled "The Historical Background of Harmonic Analysis:" 2

While the historical facts in any concrete situation rarely point a clearcut moral, it is worth while noting that the recent fertility of harmonic analysis has followed a refertilization of the field with physical ideas. It is a falsification of the history of mathematics to represent pure mathematics as a self-contained science drawing inspiration from itself alone and morally taking in its own washing. Even the most abstract ideas of the present time have something of a physical history. It is quite a tenable point of view to urge this even in such fields as that of the calculus of assemblages, whose exponents, Cantor and Zermelo, have been deeply interested in problems of statistical mechanics. Not even the influence of this theory on the theory of integration, and indirectly on the theory of Fourier series, is entirely foreign to physics. The somewhat snobbish point of view of the purely abstract mathematician would draw but little support from mathematical history. On the other hand, whenever applied mathematics has been merely a technical employment of methods already traditional and jejune, it has been very poor applied mathematics. The desideratum in mathematical as well as physical work is an attitude which is not indifferent to the extremely 
instructive nature of actual physical situations, yet which is not dominated by these to the dwarfing and paralyzing of its intellectual originality. Viewed as a whole, the theory of harmonic analysis has a very fine record of this sort. It is not a young theory, but neither is it yet in its dotage. There is much more to be learned and much more to be proved.

\section{Mathematics and the Natural Sciences}

One of the most striking features of recent developments in the physical sciences has been the convergence of focal theoretical problems with major themes in mathematical research. Mathematical concepts and tools that had arisen in an autonomous way in relatively recent research have turned out to be important components in the description of nature. At the same time, this use of novel mathematical tools in the sciences has reacted upon the development of mathematical areas having no direct connection with scientific applications to yield new and surprising mathematical consequences. We ask whether this kind of interaction will continue in a serious way in the foreseeable future, and, if so, what the consequences will be for the future development of mathematics and the sciences.

Let us begin our analysis by examining the different ways in which novel, relatively sophisticated mathematical tools have been applied in recent scientific developments. We may classify them into five relatively broad modes of attack.

(1) The use of sophisticated mathematical concepts in the formulation of new, basic physical theories on the most fundamental level. At the present moment, this takes the form of the the superstring theory, which has as its objective the total unification of all the basic physical forces and interactions-electromagnetic, weak, strong, and gravitational. This new phase of physical theory, which is the culmination of the earlier development of gauge field theories and of theories of supersymmetry, exhibits the use of a wide variety of relatively new mathematical tools developed in the past two decades; examples are Kac-Moody algebras and their representations, the existence of Einstein metrics on compact Kahlerian manifolds satisfying simple topological restrictions, and representations of exceptional Lie groups. The body of techniques and mathematical arguments embodied here includes the theory of Lie groups and algebras, their generalizations and representation theory; differential geometry in its modern global form in terms of vector bundles; the study of the ex- 
istence of solutions of manifolds of highly nonlinear partial differential equations; differential and algebraic topology; and the whole melange of analysis, algebra, and geometry on manifolds that has been called global analysis. The implementation of this program may involve still other major directions of mathematical research-for example, noncommutative geometries based on rings of operators.

A similar pattern of use of sophisticated mathematical tools in the development of fundamental physical theories appeared earlier in the context of the study of instantons in gauge field theories and the study of singularities in the equation of general relativity in connection with black holes. What must be strongly emphasized is that the role assumed by sophisticated mathematics was not the result of a willful act by either physicists or mathematicians, but of the intrinsic necessities of the development of the physical theory. Physicists, no matter how sophisticated mathematically they may be, are not free ad libitum to choose the mathematical tools they wish to use. Certainly, the mathematicians have no power to prescribe such uses to the physicists. We are very far from the decades after World War II when it was a commonplace among physicists that all the mathematics they would ever need had been completely worked out (at least as far as the involvement of research mathematicians was concerned) before World War I. It is the radical transformation of fundamental physics in the past decades that has caused the disappearance of this commonplace, and not any basic transformation in the sociology of the relations between physicists and mathematicians.

(2) A focal interest in the complex mathematical consequences of simple physical laws. One sees major examples in the modeling of turbulence in terms of bifurcation, of the asymptotic properties of differential equations, and of iteration of simple nonlinear transformations (Hopf bifurcation, the Lorenz equation, strange attractors, and Feigenbaum cascades). Simple causal mechanics can be shown to lead to disorderly regimes (chaos), but in relatively simple and classifiable forms. A historically earlier example of an attack on turbulence in the 1930s to 1950s used models in terms of stochastic processes where disorder was directly injected into the premises of the theory. Another current example is the use of fractal models (self-similarity under changes of scale, fractional Hausdorff dimensions) to describe complex phenomena in the study of materials.

(3) Mathematical models of pattern formation and symmetry breaking 
as paradigms for structured systems developing out of apparently unstructured regimes. We might think of this mode of attack (which goes back to Turing in 1952) as the converse of (2). Stable structures arise from mathematical models of differential equations or stochastic games of an apparently structureless nature in the presence of noise and possible disorder. The most striking paradigm is the oscillating chemical reaction of the Belousov-Zhabotinskii type. The objective here is eventually to model phenomena in such areas as developmental biology and brain function.

(4) Soliton theories, involving the existence in nonlinear differential equations of stable structures (solitons) arising from complete integrability. The now classical paradigm is the Korteweg-De Vries equation of shallow wave theory, rediscovered by Kruskal and his collaborators in the late 1950s after an earlier partial rediscovery in computer experiments by FermiPasta-Ulam. New models of a similar kind have been found and extensively analyzed as a possible way of describing a broad range of physical and engineering phenomena.

(5) The need to develop a usable and fruitful mathematical theory of complex systems whose elements might well be simple to an extreme but whose complexity arises from the interaction of these elements, whether linear or nonlinear, local or global. It is abundantly clear that every mode of analysis in science or in practice will eventually get to the stage where this theme is dominant.

After this summary of major themes in present-day scientific investigations to which sophisticated mathematical tools are being applied, we may ask whether this is really a new situation. A careful answer to this question demands an analysis with a historical and philosophical focus, which I present in the final section of the paper.

\section{Mathematics and the Computer}

The observant reader is already aware that the description in section 2 of the mathematical component of important themes in contemporary scientific research omitted explicit mention of the high-speed digital computer, one of the most conspicuous objects of our age. In the context of the present kind of discussion, this might seem to many like a performance of Hamlet without the Noble Dane. Yet we must segregate the discussion of the computer and its interrelation with the development of contemporary mathematics, both because of its important and distinctive role 
and because of the prevalence and intensity of myths in this domain that prevent realistic assessment of the situation.

We are all very conscious of the role of the high-speed digital computer as one of the decisive facts of the present epoch and for the foreseeable future. We all know of the tremendous impact it has had on the structure of processes in industrial society that depend on calculation, communication, and control. In practice, this excludes very few domains of human existence in modern society, whether technological, economic, social, political, or military. The sciences and mathematics have not been immune from this impact. Indeed, the scope and nature of scientific and mathematical instrumentation and practice in our society have already been radically changed by the existence of high-speed digital computation and its continual decrease in cost during recent decades. I have deliberately used the unusual phrase mathematical instrumentation to point up the radically new fact that such a phenomenon now exists and is an important component of our situation.

At the same time, although we are all conscious of the importance of the digital computer (sometimes to the point of hysteria), and indeed are inundated with advertising hyperbole from the most diverse quarters about all the wonders that supercomputers will accomplish, many are much less conscious of what is ultimately an even more important fact: the computer is as much a problem as it is a tool. We must understand the nature and limitations of this most powerful of all human tools. It is important to know what cannot be computed and the dangers of what can be miscomputed.

These limitations can be seen most plainly in the context of mathematical and scientific practice. Perhaps the most significant use of the computer in this context is as an experimental tool, sometimes even displacing the laboratory experiment altogether. One translates a scientific or mathematical problem into a simpler mathematical model and then uses the computational power of the computer to study particular cases of the general model. This approach has turned out to be very useful, particularly when the conditions for experiment in the usual sense or of precise calculation become impossibly difficult. The mystique of such practices has grown to such an extent that some speak of replacing Nature, an analog computer, by a newer and better model of a digitalized nature.

The drawbacks and dangers of such practices without a background of thorough critical analysis are equally clear. We must ask about the ade- 
quacy of the model, about the accuracy (not to say the meaningfulness) of the computational process, and, last but not least, about the representative character of the particular cases that one computes. Without serious cross-checks on these factors, we are left with yet another case of the zeroth law of the computer: garbage in, garbage out, particularly with serious scientific and mathematical problems that cannot be solved by computation as they stand. One replaces them by manageable problems, and the validity of the replacement is precisely the crucial question. It is the importance of this question that has led to pointed comments about the adjective scientific in the currently fashionable emphasis on programs for scientific computation on supercomputers.

These critical questions do not mean that we should neglect the computer as a tool in science and mathematics. They do point up a sometimes neglected fact-namely, that the computer is a difficult tool whose use must be studied and refined. Computers are brute force instruments; their effective use depends vitally on human insight and ingenuity. I intend here to emphasize the importance of the intellectual arts and insights that are or can be connected with the digital computer and its uses. These intellectual arts have a vital relation to the mathematical enterprise. They constitute a specialized and different way of applying classical mathematical ideas and techniques with radically new purposes in mind. Their vitality, both intellectual and practical, depends in an essential way upon a continuing contract with the central body of mathematical activity.

There is an interesting and slightly ironic aspect to the relationship between computer science and the central body of mathematics. Since the mid-nineteenth century, mathematicians and physical scientists have tended to see a dichotomy between mathematics that is applicable to the uses of physical modeling and calculation and another kind that is not applicable. The rules for this division have changed in recent years, with an everincreasing diversity of mathematical themes and theories falling into the first category. Even so, the stereotype tends to persist, and some areas of active mathematical research-like algebraic number theory or mathematical logic-tend to be relegated to the second category. Yet it is precisely these areas, grouped together with various forms of combinatorics under the general label of discrete mathematics, that have turned out to be most vital in major areas of advance in computer science. The basic theoretical framework of computer science and the development of complexity of computation rest upon the foundation of mathematical logic. 
The development of algorithms depends essentially upon combinatorics, number theory, and, most recently, on probabilistic models of a combinatorial type. The practical area of coding and cryptology is vitally dependent upon sharp results in number theory and algebraic number theory.

We should reject efforts to oppose the natural sciences to the "artificial" sciences. Human art and artifice are part of all the sciences, as is the confrontation with the objective realities beyond human will and control that we personify under the figure of Nature. Indeed, computer science in its necessary advance, seen today under such perspectives as parallel processing, artificial intelligence, and expert systems, and the whole family of problems subsumed under the label of computer systems and structure, can be seen as part of the general perspective of complexity of organization.

\section{The Core of Mathematics}

There is a danger in any form of discussion of the role of mathematics that emphasizes (as I have done) the active participation of new mathematical concepts and tools in the development of other scientific disciplines. Despite the strong emphasis on the new, it is far too easy to use such an approach as a prescription for future mathematicians simply to facilitate the interactions that I have described. Such a prescription would be a recipe for a massive failure, not only for the development of mathematics itself but also for the sciences. Such prescriptions are based upon the unconscious principles that creativity and newness in conceptual advance are always a matter of the past. The autonomy of mathematical research, in the sense of its freedom from any strong dependence upon the current processes of research in other disciplines and upon their rhythm of activity, has been one of the principal components of its creativity throughout its lengthy history since the Greeks. There are commonsense reasons why this creativity is important for the sciences: for example, when the advance of scientific understanding needs mathematical concepts, theories, or methods of calculation and argument, it is often essential that they be already available in a reasonably usable form.

Once mathematical problems are solved in any context, the solutions can be digested and turned to new uses in other contexts. Yet new mathematics (concepts, solutions, theorems, algorithms, proofs, calculations), if it is genuinely new, must be created by someone, and whoever 
does the job is a mathematician by definition. The task of the practitioner of another scientific discipline with respect to mathematics is to use it to understand and analyze the subject matter of that discipline, to see through the mathematics to the structure of the subject matter. From the fact that mathematics from the latter point of view ought to be transparent, one cannot draw the false conclusion that mathematics does not exist and needs no process of development in its own right.

It may seem like a paradox to some that I should introduce this strong affirmation of the essential autonomy of mathematics into a paper devoted to the interaction of mathematics and the sciences. This paradox is superficial. Any affirmation of interaction is only significant if the two sides of the interaction have a full-fledged separate existence and meaningfulness. In particular, we must affirm a central autonomous core of meaning in the mathematical enterprise if our thesis of strong interaction is to have full significance.

What is this core of meaning? I shall give a number of related answers in the form of programmatic definitions of mathematics. Each of these definitions points to important characteristics of mathematical practice, and each program leads to a slightly different perspective on that practice. It would take me too far afield to describe the interrelation of these perspectives and the tension between them. Suffice it to say that I am among those who believe in an essential unity of mathematics, though rejecting some of the dogmatic and oversimplified programs for achieving that unity by putting mathematics in a Procrustean bed and cutting off some of its limbs.

(1) Mathematics is the science of significant forms of order and relation.

(2) Mathematics is the science of the structure of possible worlds.

(3) Mathematics is the science of infinity.

(4) Mathematics is the science of the structure of complex systems.

(5) Mathematics is the study of the modeling of reality in symbolic form.

Each of these definitions taken by itself is a deep truth in the sense of Niels Bohr: its negation is also a deep truth. Taken jointly, they give us a reasonable perspective on the broad range of mathematics since the Renaissance. (Definitions 1 and 2 are due to Descartes and Leibniz, combined under the term mathesis, whereas definition 3, which was originated by Leibniz, was revived in modern times by Poincaré and Weyl.) 


\section{Applicable Mathematics}

Mathematical research in its various forms is an enterprise of great vitality in the present-day world (although it is invisible to some outsiders). Despite its fundamental autonomy, the enterprise of advanced mathematical research has interacted strongly in the last two decades with various advances in the sciences. For the purposes of the present discussion, I present two kinds of evidence.

The first consists of taking a conventional breakdown of the principal active branches of contemporary mathematical research and inquiring in general terms whether these branches have interactions of the type described with the sciences. In the table of organization for the International Congress of Mathematicians in Berkeley, California, in the summer of 1986, we have such a breakdown in the division of the Congress into nineteen sections. Of these nineteen sections, we may set aside two (history of mathematics, teaching of mathematics) and ask about the applicability of the seventeen mathematical areas in this classification. Five (probability and mathematical statistics, mathematical physics, numerical methods and computing, mathematical aspects of computer science, applications of mathematics to nonphysical sciences) relate directly to the sciences and technology. Eight have direct relation in contemporary practice to theory and practice in the natural sciences (geometry, topology, algebraic geometry, complex analysis, Lie groups and representations, real and functional analysis, partial differential equations, ordinary differential equations and dynamical systems). The remaining four (mathematical logic and foundations, algebra, number theory, discrete mathematics and combinatorics) have an equally vital relation to computer science. There is no residue of mathematics that is fundamentally not applicable on this list.

The second kind of evidence is illustrated by the study of the soliton theory of the Korteweg-De Vries equation in the periodic case. The applications of algebraic geometry and complex analysis to the study of the Korteweg-De Vries equation under periodic boundary conditions not only contributed to the understanding of the physical model involved but reacted upon the disciplines involved. New ideas and methods in both mathematical disciplines arose from this interaction, resulting in the solution of classical problems in algebraic geometry and function theory. In an even more striking case, the young Oxford mathematician Simon Donald- 
son observed that if one combined the mathematical techniques developed for the study of the mathematical theory of gauge fields by Karen Uhlenbeck with the penetrating geometric attack upon the structure of four dimensional manifolds of Michael Freedman one could obtain a new and totally surprising geometric result in four dimensions. The result in question asserts that, unlike Euclidean spaces in every other dimension, fourdimensional Euclidean space possesses two systems of coordinates that are fundamentally different from one another.

These two cases illustrate a possibility that has turned into a current reality - that the strong mathematical attack upon mathematical problems raised in the context of development of scientific research can provide the occasion and stimulus for major conceptual advances in mathematics itself.

\section{Historical Perspectives}

To close, let us turn to the question of the future relation of mathematics and the sciences. We may recall another well-known saying of Niels Bohr: Prediction is difficult, especially of the future. Attempts to predict the future are hypotheses about the past and present. Let us formulate a hypothesis that we can check for coherence and accuracy against the past and present and then try to gauge its consequences for the future.

Our scientific tradition is inherited from the civilization of the ancient Greeks. It was there that the concept of science as a self-conscious structuring of objective lawful knowledge of the world (or, more strictly, of the hidden processes of the world) first arose. Although the Greeks investigated the full range of human experience, their achievement in creating permanent scientific knowledge was primarily in the mathematical sciences, in mathematics itself and in such highly mathematical disciplines as mathematical planetary astronomy, musical theory, and the mathematical treatment of statics. The Greeks created a highly perfected form of sophisticated mathematical theory treating of whole number, geometry, ratio, and geometrical measure. In this theory, they also perfected a fully mature concept of mathematical argument, of logical deduction. On the basis of these achievements, Plato might argue in his celebrated dialogue Timaeus for a mathematical myth of the cosmos and its formation on the basis of geometric elements, and Aristotle could formulate the logical principles of deduction while rejecting the possibility of mathematical laws for the phenomena of terrestrial physics.

It is fashionable to talk of scientific revolutions. On the most fundamental level, there has been only one scientific revolution-that of the seven- 
teenth century, in which modern science was formed. The concept of science that this century produced gave a description of the cosmos, the physical universe, in terms of the geometry of space and numerical relations, a description that applied to both the skies and the earth. It saw this cosmos as a realm of objective lawful relations, devoid of human agency or affect. Reality was separated after Descartes into two completely distinct parts, the physical universe and a separate world of human consciousness and spirit. In this framework, it made total sense for human consciousness to try to determine the secrets of natural processes not by passive observation but by transforming nature by experiment.

There was a mathematical counterpart of the new physical science, which served both as its precursor and principal tool. This was the mathematics of the new algebra and of the analytic movement of Vieta and Descartes, a mathematics that substituted calculation and manipulation of symbolic expressions for the deductive sophistication of the Greeks. It substituted the analysis of complex phenomena into simple elements for the Greek emphasis on deduction. In the seventeenth century, this new mathematics had two overwhelming triumphs. The first was the creation of an analytic geometry through which the geometric structure of space could be transformed by coordinatization into the subject matter of algebraic analysis. The second was the invention of the great analytic engine of the differential and integral calculus, by which the sophisticated and difficult arguments by exhaustion of Eudoxos and Archimedes for handling infinite processes were replaced by much simpler and more manageable algebraic formulae or calculi. This was the tool with which Newton built his great mathematical world-machine, the central paradigm for the scientific world pictures of all succeeding ages.

There are essentially two forms in which objective human knowledge can be formulated: in words and in mathematical forms. Aristotle opted for the first and created a systematic description of the world in which the subject-predicate form of the sentence was transformed into the pattern of the individual object or substance possessing a certain quality. From the seventeenth century on, modern science has rejected this form of description and replaced it by descriptions in various mathematical forms. These forms have altered as the stock of mathematical forms has increased and become richer and more sophisticated. The original forms were geometric, in the style of the Greeks. In the Renaissance, a new and more flexible concept of number, the 'real' number in the present-day sense, came into being as the common measure of lengths, areas, volumes, 
masses, and so forth, without the precise distinction between these measures in terms of geometric form to which the Greeks had held. In the ensuing development of algebra, new kinds of 'numbers' appeared as the solutions of algebraic equations. Since they were not numbers in the old sense, some were called 'imaginary', and mixtures of the two types were called 'complex'. It was not until the end of the eighteenth century that these 'complex' numbers were fully naturalized as members of the commonsense mathematical realm by being identified in a simple way with the points of a Euclidean plane, the complex plane.

Since the seventeenth century, the enterprise of the scientific description of nature has continued to develop within this mathematical medium, which was dimly foreshadowed by Plato's mathematical world myth. New scientific disciplines developed, and they entered the same framework of numerical relationship, geometric form in space, and formulation of basic principles in mathematically expressed laws. As Kant put it in a well-known aphorism from his Metaphysical Foundations of Natural Sciences: "In every special doctrine of nature, only so much science proper can be found as there is mathematics in it."

In the nearly four centuries that have elapsed since Galileo began the seventeenth century scientific revolution, the curious relationship of autonomy and mutual dependence between the natural sciences and mathematics has taken ever more complex and sophisticated forms. The mathematical medium in which the various sciences live has continued to develop and take on new shapes. In the early nineteenth century, the intuitive concept of symmetry that was applied to the study of the roots of algebraic equations gave rise to the concept of group. The group concept, passing through the medium of its application to geometry and differential equations, became in the twentieth century the most essential building block of the fundamental description of the physical universe. The concept of space, enriched by the insights of Gauss and Riemann, gave rise to the richer geometric concepts of Riemannian manifold and of curvature, through which the theory of general relativity of Einstein was to describe the cosmos. Through the analysis of integral equations and differential equations in the early twentieth century, the concept of an infinite-dimensional vector space was born, and the especially rich concept of a Hilbert space. Hermitian operators on a Hilbert space with their spectral theory served as the eventual underpinning of the the formal structure of quantum mechanics. These are three important examples of a very broad phenomenon. 
New concepts and theories arise in mathematical research through the pressure of the need to solve problems and create intellectual tools through which already existing mathematical theories and structures can be extended and applied. Once the new concepts and theories are established, they themselves become the focus of intensive investigation. The new is achieved by mathematical imagination, applied through the medium of mathematical constructions through which the new concepts and structures are given definite form. Although the imaginative process is free in some ultimate sense, its result once produced becomes a new objective realm of relationship of a determinate character. Classical tools like deduction and calculation are used to establish its properties, leading to new technical problems that may eventually demand new concepts and constructions for their solution. The jump of insight and imagination that leads to new mathematical breakthroughs belies the stereotypes of mathematical activity as an automatic, machinelike process of mechanical application of formal rules.

Mathematical research as a whole balances the radical process of generation of new concepts and theories with the conservative tendency to maintain in existence all those domains, problems, and conceptual themes that once became established as foci of significant mathematical research. The balance between these opposing tendencies gives rise to the striking fact that, at the same moment, one can find active research programs of apparently equal vitality bearing on two themes, one of which is two thousand years old and the other perhaps only a decade old. Yet the two-thousandyear-old problem might well be solved with tools and concepts of relatively recent vintage.

The richer the repertoire of modern mathematical research, the broader the arsenal of concepts and tools available for the use of the mathematicized sciences. The difficulty lies in the problem of communication, of the scientific practitioners being able to penetrate through the difficulties of translation between the languages of different disciplines, of knowing what is relevant in the concepts and techniques that are available.

As the concerns and principal foci of scientific interest move into domains ever further from the classical ones of theory and experience, the role of mathematical ideas and techniques inevitably grows because they often provide the only tools by which one can probe further into the unknown. This is particularly true for domains involving complexity of organization or nonlinearity of interaction, the future frontier of the major themes of scientific advance. Although they may become the subject 
matter of major scientific disciplines in their own right, I doubt that this will lead to the disappearance of professional differences between specialists in various disciplines in attacking these scientific problems. The difference between specialties has a positive function as well as negative consequences. Specialists can rely upon the intellectual traditions and resources of their scientific specialty, and this applies with the greatest force to the mathematician. We can ask for a broader and more effective effort at communication, however, among those concerned with common problems, and we can cultivate an active interest in and sympathy with the thematic concerns of other specialties than our own.

\section{Notes}

1. In The Works of the Mind, ed. Heywood and Nef (Chicago: University of Chicago Press, 1945); reprinted in The World of Mathematics, ed. J. Newman, vol. 4 (New York: Simon and Schuster, 1956), pp. 2053-63.

2. In Semi-Centennial Addresses of the American Mathematical Society, 1938. 